\title{
МОДЕЛИРОВАНИЕ КОНЦЕНТРАЦИОННЫХ ПРЕДЕЛОВ НА ОСНОВЕ НЕЙРОННЫХ СЕТЕЙ
}

\author{
Осипов А.Л., Трушина В.П., Павлик И.О.
}

Изучаются модели прогнозирования концентрационных пределов с использованием нейросетевых технологий. Разработано программное обеспечение для реализации этих моделей. Показана эффективность работы системы на экспериментальном материале.

Ключевые слова: моделирование; нейронные сети; принятие решений; программирование; концентрационные предель; выборка; структурная формула.

\section{MODELLING OF CONCENTRATION LIMITS BASED ON NEURAL NETWORKS}

Osipov A.L., Trushina V.P., Pavlik I.O.

We study the forecasting model with the concentration limits is-the use of neural network technology. The software for the implementation of these models. It is shown that the efficiency of the system in the experimental material.

Keywords: modeling; neural networks; decision-making; programming; concentration limits; sampling; structural formula.

Для проведения эффективных мероприятий по обеспечению пожаровзрывобезопасности, а также создания безопасных условий труда необходимо правильно и полно оценивать пожаровзрывоопасные и физико-химические свойства исследуемых соедине- 
ний, таких как адиабатическая температура горения, температура вспышки, верхний и нижний концентрационные пределы воспламенения и др. [4, с. 41]. Зачастую сведения по пожаровзрывоопасности новых или еще только синтезируемых химических веществах отсутствуют. В связи с тем, что экспериментальное определение показателей пожаровзрывоопасности сопряжено с трудоемкими исследованиями, в настоящее время единственным способом, который удовлетворяет возрастающую потребность в данных о пожаровзрывоопасности, являются расчетные методы, которые требуют эффективных математических моделей. Расчетные методы полезны как для прогнозной оценки пожаровзрывоопасности используемых веществ, так и на этапе проектных исследований изза необходимости оценки влияния возможных колебаний составов реакционных смесей в химико-технологических процессах.

В последние несколько лет наблюдается большой интерес к нейронным сетям. Нейронные сети являются наиболее эффективным инструментом для задач прогнозирования, применяемому практически в любой ситуации, когда имеется связь между переменными-предикторами (входами) и прогнозируемыми переменными (выходами), даже если эта связь имеет очень сложную природу и ее трудно выразить в обычных терминах корреляций или различий между группами.

Концентрационный предел распространения пламени (КПР) одно из значимых пожаровзрывоопасных свойств веществ, определение которого является необходимым действием при определении категории помещений и зданий по взрывопожарной и пожарной опасности, при разработке мер по предотвращению пожаров и взрывов в технологическом оборудовании и т.д.

Концентрационный предел распространения пламени (воспламенения) (КПВ) - минимальное/ максимальное содержание горючего вещества в однородной смеси с окислительной средой (окислителем), при котором возможно распространение пламени 
по смеси на любое расстояние от источника зажигания. По максимальному и минимальному содержанию горючего в воздухе различают соответственно верхний концентрационный предел воспламенения (ВКПВ) и нижний концентрационный предел воспламенения (НКПВ). Между ними находится интервал (область воспламенения), характеризующий пожарную опасность газов, паров, пылей, жидкостей. Содержание горючего в воздухе (размерность КПР) может выражаться в \% (по объему) или в г· ${ }^{3}$ [1, с. 27].

Экспериментальный способ определения пожаровзрывоопасных свойств, в том числе и определение НКПВ, достаточно затратен и трудоемок [1, с. 35]. Намного эффективнее и удобнее является использование компьютерных программ, реализующие наиболее точные алгоритмы и математические модели расчета таких показателей [3, с. 89]. Разработка программных средств в целях предсказания пожаровзрывоопасных свойств химических веществ на основе нейросетевых технологий является актуальной задачей.

Для прогнозирования НКПВ отобрана информация о 300 соединениях, относящиеся к различным классам химических веществ: наименование вещества, класс, структурная формула, значение НКПВ [1, с. 57; 2, с. 65].

Задача прогнозирования решается путем обучения нейронной сети выборкой из химических соединений с заданным показателем НКПВ. Имея готовую нейронную сеть, можно ввести структурную формулу вещества и получить предсказанное значение НКПВ для химического соединения. Программа также позволяет вносить в базу данных информацию о новых веществах, значения НКПВ которых достоверно подтверждены. С учетом этих новых данных имеется возможность переобучения нейронной сети, а также экспортировать отчеты по результатам прогнозирования в MS Excel.

На синапсы нейронов первого слоя подается информация о наличии и количестве дескрипторов, присутствующих в структурной формуле. В качестве таких дескрипторов отобраны сле- 
дующие фрагментарные составляющие формулы: атомы с учетом валентного состояния или атом-связь-атом. Таким образом, на входы каждого нейрона первого слоя подается следующая информация: 0 - если соответствующий фрагмент отсутствует в структурной формуле, и в случае наличия фрагмента в формуле цифра, показывающая их количество.

Для того, чтобы программа могла прогнозировать нижний концентрационный предел воспламенения необходимо обучение нейронной сети. Это обучение происходит с помощью алгоритма обратного распространения ошибки.

Алгоритм обратного распространения ошибки является одним из методов обучения многослойных нейронных сетей прямого распространения, называемых также многослойными персептронами. Многослойные персептроны успешно применяются для решения многих сложных задач.

В качестве входных данных используются готовые соединения и их коэффициенты воспламенения, полученные экспериментальным путем.

Общий алгоритм обучения представлен ниже:

1. Инициализировать веса маленькими случайными значениями.

2. Подать обучающую выборку на вход сети и подсчитать выходы каждого узла.

3. Для выходного слоя рассчитывается

$$
\delta_{k}=o_{k}\left(1-o_{k}\right)\left(t_{k}-o_{k}\right),
$$

где $\delta$ - производная выходного слоя; $o_{k}-$ выходной сигнал, $t_{k}-$ предполагаемый выходной сигнал.

4. Для каждого слоя, начиная с предпоследнего вычислить

$$
\delta_{j}=o_{j}\left(1-o_{j}\right) \sum_{k \in \operatorname{Children}(j)} \delta_{k} w_{j, k}
$$

где $\delta$ - производная промежуточных слоев; $o_{j}-$ выходной сигнал слоя; $w_{j, k}-$ вес слоя. 
5. Далее происходит подстройка весов

$$
\Delta w_{i, j}=\alpha \Delta w_{i, j}+(1-\alpha) \eta \delta_{j} o_{i},
$$

где $w_{i, j}$ - вес слоя, $\alpha$ - произвольная константа, $\eta$ - множитель, задающий скорость «движения», $\delta$ - производная промежуточных слоев, $o_{j}-$ выходной сигнал слоя.

где $w_{i, j}-$ вес слоя, $w_{i, j}-$ градиент.

$$
w_{i, j}=w_{i, j}+\alpha \Delta w_{i, j}
$$

6. Повторить обучение с 2 шага, если существуют еще обучающие выборки.

Так же нужно отметить, что нейронная сеть в описываемой программе использует сигмоидальную функцию активации, что позволяет более точно определять прогнозируемое значение. В качестве примера сигмоидальных функций можно привести логистическую (1) и гиперболический тангенс (2):

$$
Y=\frac{1}{1+\exp (-\alpha Y)},
$$

где $Y$ - выходной сигнал слоя, $\alpha$ - параметр наклона сигмоидальной функции активации.

$$
Y=\operatorname{th}\left(\frac{Y}{\alpha}\right)
$$

где $Y$ - выходной сигнал слоя, $\alpha$ - параметр наклона сигмоидальной функции активации. В представленной нейронной сети использован гиперболический тангенс. На рис. 1 представлена блок-схема обучения по алгоритму обратного распространения ошибки.

Сведения о веществах хранятся в файле chemicals.yml. YAMLчеловекочитаемый формат сериализации данных, концептуально близкий к языкам разметки, но ориентированный на удобство ввода-вывода типичных структур данных многих языков программирования. Непосредственно в файле chemicals.yml xpa- 
нится информация о наименованиях химических соединений, фрагментарных элементах, которые они содержат, их связей и дескрипторы этих соединений, а также Smiles-формулы. При запуске программы открывается основное диалоговое окно, содержащее следующие элементы (рис. 2):

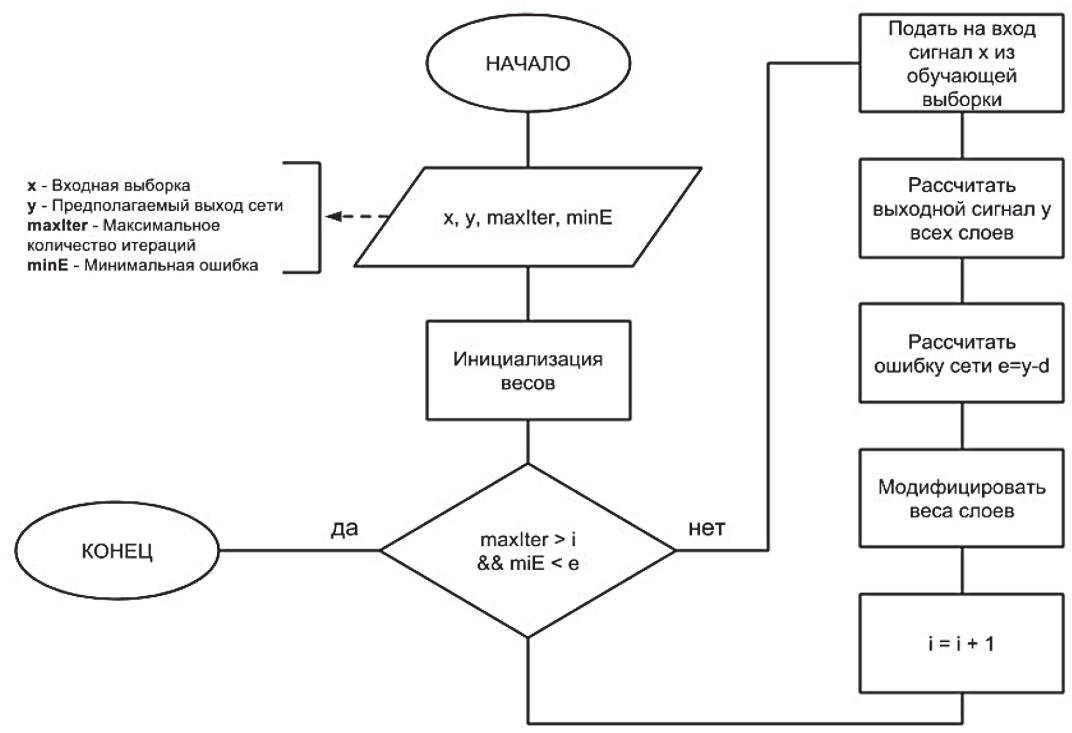

Рис. 1. Алгоритм обучения сети

- молекулярный редактор, позволяющий строить структурные формулы исследуемого соединения либо путем перетаскивания на рабочую область с панели элементов соответствующие элементы и соединять их связями, либо по Smiles-формулам;

- справочные окна, показывающие информацию о содержащихся в формуле дескрипторах (наличие и количество) и значение НКПВ;

- пункты меню «Файл» - содержащие стандартные команды работы с программой; 
- пункты меню «Сервис»: Соединения - позволяет выбрать соединения из базы данных для исследования, Обучение позволяет открыть соответствующие диалоговые окна для обучения нейронной сети, Отчет - для экспорта результатов обучения в MS Excel; Экзамен - проверка качества прогнозирования на экзаменационной выборке;

- кнопка, вызывающая диалоговое окно для добавления нового соединения в базу данных.

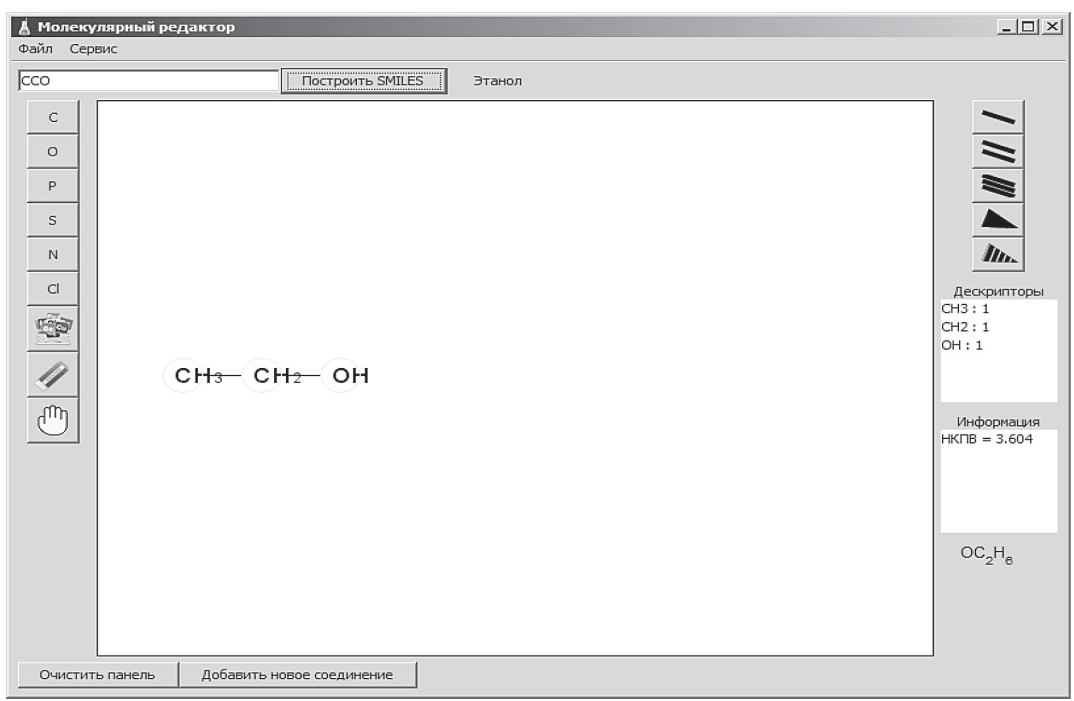

Рис. 2. Молекулярный редактор

Диалоговое окно для добавления нового вещества в базу данных содержит поля: Наименование, Smiles-формула и коэффициент НКПВ (рис. 3).

Для начала работы программы необходимо ввести структурную формулу химического соединения одним из трех способов: либо построить в молекулярном редакторе, используя панель инструментов, либо выбрать уже готовое соединение из списка, либо ввести формулу в формате SMILES в текстовое поле в верх- 
ней части экрана. Программа проверяет информацию о вводимом химическом веществе на наличие его в базе данных. Если сведения о таком веществе уже хранятся в ней, то автоматически выводится его название, в противном случае существует возможность добавления информации о веществе в базу данных: название, SMILES-формула, значение НКПВ.

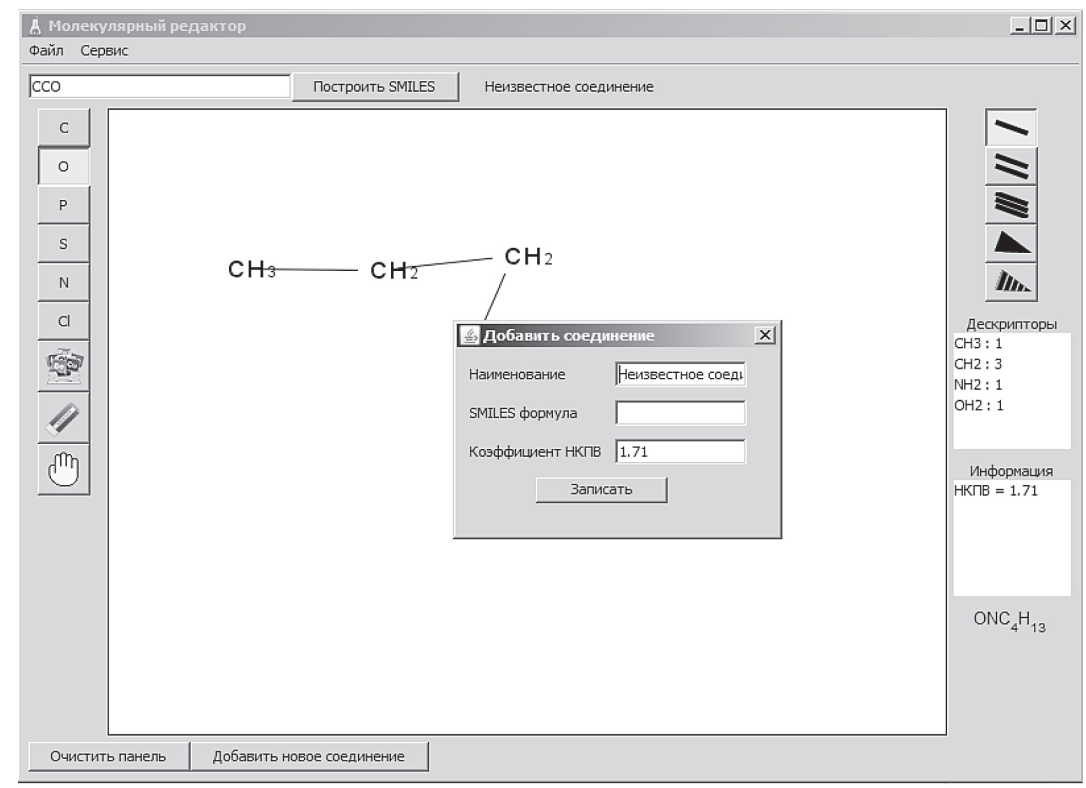

Рис. 3. Добавление нового вещества в базу данных

После ввода структурной формулы программой подсчитываются дескрипторы: в соответствующем окне показывается их наличие и количество.

После подсчета дескрипторов программа приступает к расчету коэффициента НКПВ. Для этого подгружается обученная нейронная сеть, и в нее загружаются уже готовые дескрипторы. Когда сеть подстроит значения согласно весам, она выдаст рассчитанное значение НКПВ. 
Обучение сети можно провести, выбрав пункт меню Сервис Обучение. Окно Обучение представляет собой таблицу, в столбцах которой содержится информация о наличии и количестве дескрипторов соединения и значении НКПВ, подтвержденным надежными источниками (рис. 4). По строкам таблицы расположены сведения о конкретном соединении из базы данных.

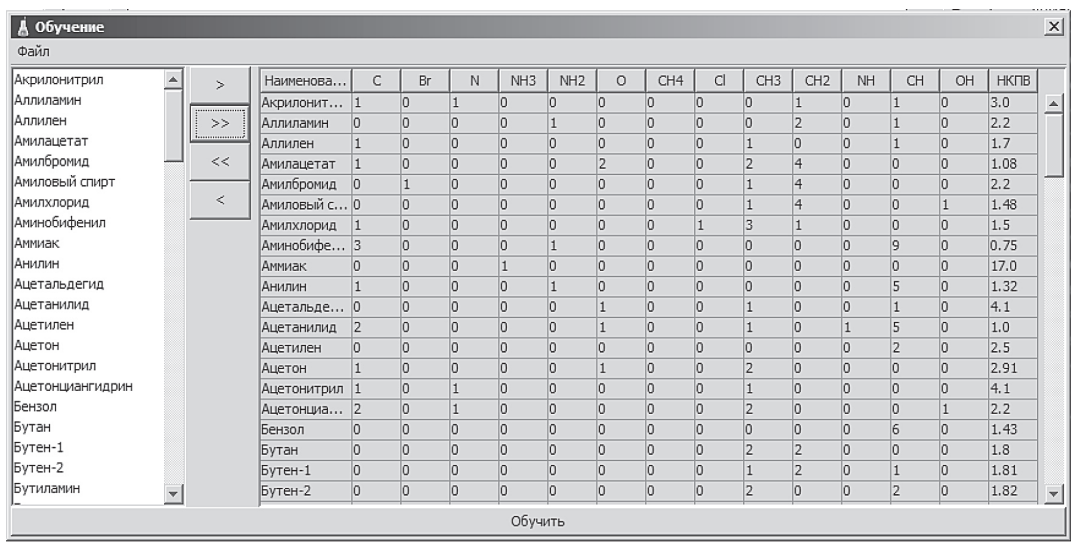

Рис. 4. Окно «Обучение»

Обучение нейронной сети начинается после нажатия кнопки «Обучить».

Для демонстрации работы программы из базы химических соединений выбран случайным образом Гептан (рис. 5).

В окне молекулярного редактора отобразились его структурная формула, наличие и количество дескрипторов, входящих в формулу, а также значение коэффициента НКПВ (рис. 6).

В ходе исследований осуществлена возможность прогнозирования нижнего и верхнего концентрационных пределов воспламенения, добавления в базу данных веществ с известными значениями НКПВ и ВКПВ, переобучение нейронной сети с учетом этих значений, а также экспорт отчетов по результатам прогнозирования в MS Excel. 


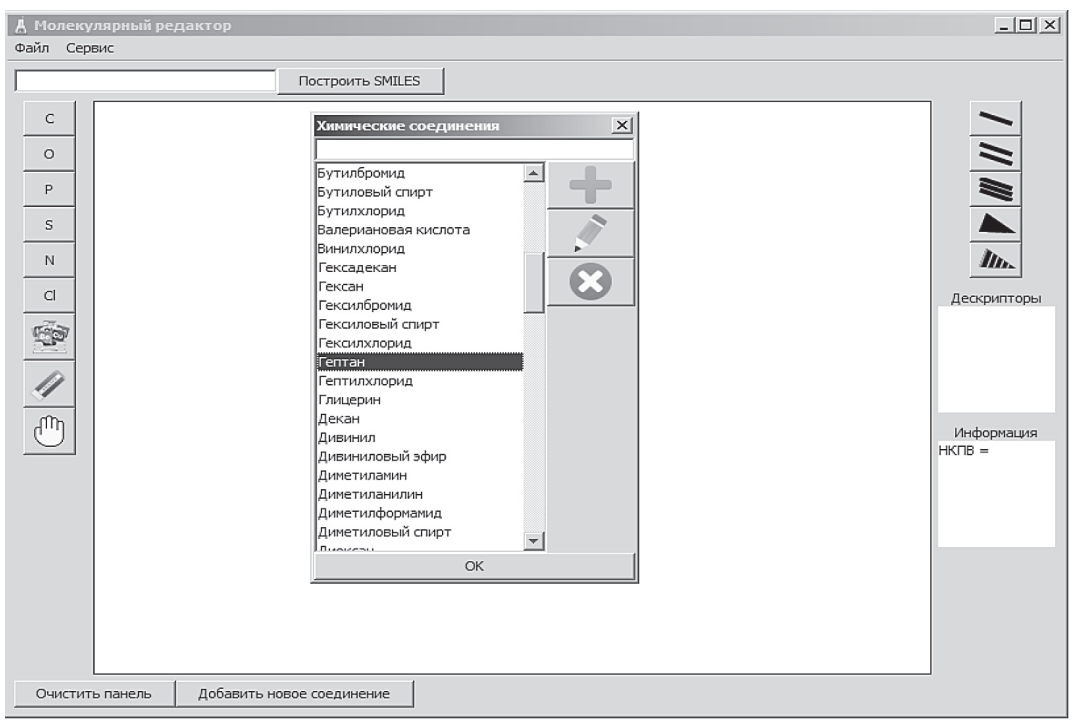

Рис. 5. Выбор соединения для прогнозирования НКВП

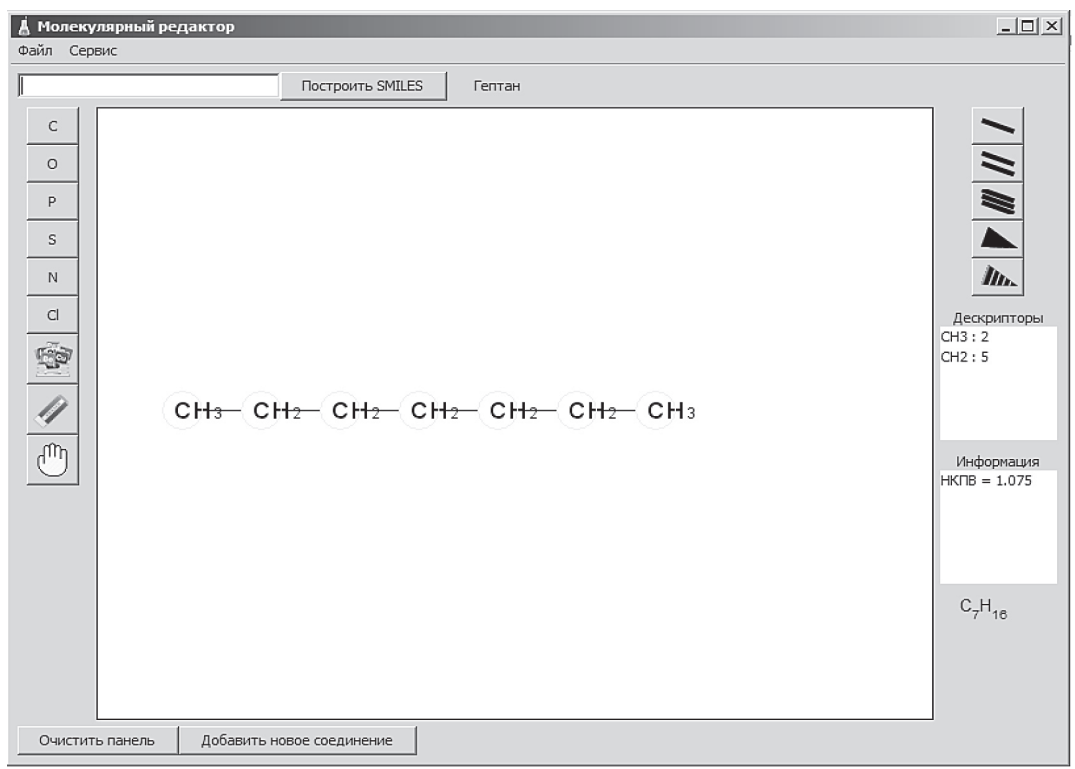

Рис. 6. Структурная формула выбранного соединения 
В ходе проведенных экспериментов были получены следующие результаты: при обучении и тестировании нейроной сети на экзаменационных выборках выдаются результаты, имеющие точность, адекватную точности экспериментального определения НКПВ и ВКПВ. Таким образом, можно сделать вывод, что программа пригодна для прогнозирования НКПВ и ВКПВ широкого класса органических соединений, и это позволяет рекомендовать ее для практического использования.

\section{Список литературы}

1. Корольченко А.Я., Крольченко Д.А. Пожаровзрывоопасность веществ и материалов и средства их тушения: справочник: в 2ч. М.: Асс. «Пожнаука», 2004. Ч.1. 713 с.

2. Корольченко А.Я., Крольченко Д.А. Пожаровзрывоопасность веществ и материалов и средства их тушения: справочник: в 2ч. М.: Асс. «Пожнаука», 2004 . Ч.2. 774 с.

3. Криветченко О.В., Павлик И.О. Компьютерная система прогнозирования нижнего концентрационного предела воспламенения //Информационно-телекоммуникационные системы и технологии (ИТСиТ-2014): Материалы Всероссийской научно-практической конференции, г. Кемерово, 16-17 октября 2014 г.; Кузбас. гос. техн. ун-т им. Т.Ф. Горбачева. Кемерово, 2014. C. $88-89$.

4. Осипов А.Л., Криветченко О.В. Компьютерная оценка нижнего концентрационного предела воспламенения химических веществ // В мире научных открытий. Красноярск: Научно-инновационный центр, 2013. №10.1(46) (Математика. Механика. Информатика). С. $34-45$.

\section{References}

1. Korolchenko A.Ya., Krolchenko D.A. Pozharovzryvoopasnost'veshchestv $i$ materialov $i$ sredstva ikh tusheniya [Fire hazard substances 
and materials and their extinguishing agents]. M.: Ass. «Pozhnauka», 2004. Part 1. 713 p.

2. Korolchenko A.Ya., Krolchenko D.A. Pozharovzryvoopasnost'veshchestv $i$ materialov $i$ sredstva ikh tusheniya [Fire hazard substances and materials and their extinguishing agents]. M.: Ass. "Pozhnauka", 2004. Part 2. 774 p.

3. Krivetchenko O.V., Pavlik I.O. Komp'yuternaya sistema prognozirovaniya nizhnego kontsentratsionnogo predela vosplameneniya [The computer system of forecasting lower flammable limit // Information and Telecommunication Systems and Technologies]. Informatsionno-telekommunikatsionnye sistemy i tekhnologii (ITSiT-2014): Materialy Vserossiyskoy nauchno-prakticheskoy konferentsii, g. Kemerovo, 16-17 oktyabrya 2014 [Proceedings of the All-Russian scientific-practical conference, Mr. Ke-mers, on October 16-17, 2014]; Kuzbas. gos. tekhn. un-t im. T.F. Gorbacheva. Kemerovo, 2014, pp. 88-89.

4. Osipov A.L., Krivetchenko O.V. V mire nauchnykh otkrytiy. Krasnoyarsk, 2013. №10.1(46), pp. 34-45.

\section{ДАННЫЕ ОБ АВТОРАХ}

\section{Осипов А.Л., Трушина В.П., Павлик И.О.}

Новосибирский государственный университет экономики и управления

ул. Каменская 52/1, г. Новосибирск, 630099, Российская Федерачия alosip@mail.ru

\section{DATA ABOUT THE AUTHORS}

\section{Osipov A.L., Trushina V.P., Pavlik I.O.}

Novosibirsk State University of Economics and Management 52/1, Kamenskaya Str., Novosibirsk, 630099, Russian Federation

alosip@mail.ru 